\title{
Targeted MAPK Pathway Inhibitors in Patients With Disseminated Pilocytic Astrocytomas
}

\author{
Anastasia Drobysheva, MD, MSc ${ }^{\mathrm{a}, \mathrm{b}}$; Laura J. Klesse, MD, PhD ${ }^{\mathrm{c}, \mathrm{d}}$; Daniel C. Bowers, MD ${ }^{\mathrm{c}, \mathrm{d}}$; \\ Veena Rajaram, MDª, Dinesh Rakheja, MD ${ }^{\mathrm{a}, \mathrm{b}, \mathrm{c}}$; Charles F. Timmons, MD, PhD ${ }^{\mathrm{a}, \mathrm{b}}$; Jason Wang, MD ${ }^{\mathrm{a}, \mathrm{b}}$; \\ Korgun Koral, MD ${ }^{\mathrm{e}, \mathrm{f}}$; Lynn Gargan, PhDd Erica Ramos, BS ${ }^{\mathrm{b}}$; and Jason Y. Park, MD, PhD ${ }^{\mathrm{a}, \mathrm{b}, \mathrm{g}}$
}

\section{Abstract}

This report presents a series of 5 pediatric patients with disseminated pilocytic astrocytomas and frequent nonfusion activating mutations Genetic variants in these patients' tumors include BRAF p.Val600Glu, BRAF p.Val600Asp, and KRAS p.Gly60_GIn62ins7. The 2 patients with $B R A F$-mutated tumors were treated with dabrafenib or a combination of dabrafenib plus trametinib. The patients had either near complete resolution of the primary tumor (BRAF p.Val600Glu) or a stable primary tumor (BRAF p.Val600Asp). Both patients showed improvement in leptomeningeal dissemination without significant toxicity. Genomic testing of disseminated pilocytic astrocytomas, particularly those arising at extracerebellar locations, may result in the identification of mutations associated with ERK/MAPK activation. Patients with these activating mutations may benefit from targeted therapies.

J Natl Compr Canc Netw 2017;15(8):978-982 doi:10.6004/jnccn.2017.0139

Pilocytic astrocytomas (PAs) are low-grade gliomas that constitute approximately $20 \%$ of all pediatric central nervous system (CNS) tumors. ${ }^{1}$ PAs in children are often associated with genetic alterations, which result in constitutive activation of BRAF or the downstream MAPK pathway. ${ }^{2}$ The most common activating events in PAs are the fusion of KIAA1549 and BRAF resulting in an activated oncogene $(65 \%-90 \%), 3,4$ and a single nucleotide variant in BRAF resulting in a valine-toglutamate substitution in amino acid codon 600 (BRAF p.Val600Glu) (6\%-9\%)., BRAF p.Val600Glu is frequently observed in other low-grade gliomas, including pleomorphic xanthoastrocytomas $(50 \%-66 \%)$ and gangliogliomas (20\%). ${ }^{6}$

From the aDepartment of Pathology, University of Texas Southwestern Medical Center; 'Department of Pathology and Laboratory Medicine, Children's Health; 'Department of Pediatrics, University of Texas Southwestern Medical Center; 'Department of Pediatrics, Children's Health; 'Department of Radiology, University of Texas Southwestern Medical Center; 'Department of Radiology, Children's Health; and ${ }^{9}$ Eugene McDermott Center for Human Growth and Development, University of Texas Southwestern Medical Center, Dallas, Texas.
Patients with PAs typically have 10-year survival rates of $80 \%$ to $100 \%$ with complete resection. However, tumors located along the midline and disseminated PAs have a suboptimal response to conventional chemotherapy and a relatively poor prognosis. ${ }^{7}$ There are several reports of successful treatment of low- and high-grade gliomas with BRAF inhibitors (vemurafenib and dabrafenib). ${ }^{8-13}$ There is only a single report of 2 patients with PAs who were treated with trametinib, a MAPK pathway inhibitor, ${ }^{5}$ and no reports on MAPK pathway-targeted therapy for disseminated PAs. This report presents a series of 5 patients with progressive disseminated PA, and discusses the clinical response to targeted molecular therapy in 2 patients.

Submitted March 9, 2017; accepted for publication May 8, 2017 The authors have disclosed that they have no financial interests, arrangements, affiliations, or commercial interests with the manufacturers of any products discussed in this article or their competitors.

Correspondence: Jason Y. Park, MD, PhD, Department of Pathology and Laboratory Medicine, Children's Medical Center Dallas, 1935 Medical District Drive, Dallas, TX 75235. E-mail: jaspar@childrens.com 


\section{Case Presentations}

Five pediatric patients ( 3 females, 2 males; median age, 5 years; age range, $1-15$ years) with disseminated PAs were recently treated at our medical center (2015-2016; Table 1); 3 had spinal leptomeningeal dissemination at the time of analysis. As part of clinical care, the patients' formalin-fixed paraffinembedded tumors were analyzed with clinical nextgeneration sequencing tests using either an in-house test ( 25 genes) or a commercial reference laboratory (315 genes; Foundation Medicine, Cambridge, MA). Both DNA sequencing tests examined known activating mutations of BRAF, KRAS, NRAS, and PIK3CA; however, KIAA1549-BRAF fusion testing was only part of the 315-gene test. Four patients had an MAPK pathway-activating variant: BRAF c.1799T >A, p.Val600Glu (V600E) $(n=2)$, BRAF c.1799_1800delinsAT, p.Val600Asp (V600D) $(\mathrm{n}=1)$, and KRAS c.181_182ins21, p.Gly60_Gln62ins7 (G60_Q61ins7) (n=1). The fifth patient's tumor was negative for activating variants by the 25 gene test. Four patients were treated with radiotherapy (RT) and/or conventional chemotherapy with variable clinical results (Table 1). Two patients (with BRAF V600E or V600D) had progressive disease and were treated with MAPK pathway inhibitors.
Patient 1 was diagnosed with PA at 5 years of age when she presented with persistent headache; MRI showed a solid and cystic posterior fossa tumor with patchy contrast enhancement. Over the next 7 years she underwent 3 incomplete surgical resections before experiencing progression with persistent growth of residual tumor and leptomeningeal dissemination. The 315-gene test identified BRAF V600E in her tumor; she was started on dabrafenib at $75 \mathrm{mg}$ twice daily. After 3 months of therapy, the patient had a near complete response with mild T2 signal at the resection site, and resolution of leptomeningeal dissemination. Her most recent MRI, performed at 12 months after treatment initiation, showed minimal residual abnormal signal at the primary tumor site. Skin rash was the only toxicity observed.

Patient 2 presented with nystagmus at 13 months of age. Imaging identified a large optic-hypothalamic PA. She underwent surgical debulking and received 4 distinct chemotherapy regimens over 3 years (carboplatin/vincristine, cisplatin/cyclophosphamide/ etoposide, bevacizumab/irinotecan, temozolomide/ everolimus) due to progressive disease (Figure 1A-D). BRAF V600D was identified in the tumor by the 25 -gene test, and she was treated with dabrafenib at $50 \mathrm{mg}$ daily due to further progression. After 3 months of therapy, there was improvement of

\begin{tabular}{|c|c|c|c|c|c|}
\hline & Patient 1 & Patient 2 & Patient 3 & Patient 4 & Patient 5 \\
\hline Age at diagnosis, y & 5 & 1 & 4 & 7 & 15 \\
\hline Sex & Female & Female & Male & Male & Male \\
\hline Ethnicity & Asian & Hispanic & White & White & White \\
\hline $\begin{array}{l}\text { Primary tumor } \\
\text { location }\end{array}$ & Posterior fossa tumor & $\begin{array}{l}\text { Optic-hypothalamic } \\
\text { tumor }\end{array}$ & Midbrain tumor & Optic-thalamic tumor & Third ventricle tumor \\
\hline Standard therapy & Surgical resections, $x 3$ & $\begin{array}{l}\text { Surgical resection, } x 1 \\
4 \text { different CMT } \\
\text { regimens }\end{array}$ & ETV, CMT, RT & CMT & $\begin{array}{l}\text { Surgical resection, } x 1 \\
\text { CMT } \\
\text { RT }\end{array}$ \\
\hline $\begin{array}{l}\text { Response to } \\
\text { standard therapy }\end{array}$ & $\begin{array}{l}\text { PD at primary site } \\
\text { and new metastatic } \\
\text { disease }\end{array}$ & $\begin{array}{l}\text { PD at primary site } \\
\text { and new metastatic } \\
\text { disease }\end{array}$ & $\begin{array}{l}\text { SD with extensive } \\
\text { metastatic } \\
\text { dissemination }\end{array}$ & $\begin{array}{l}\text { SD with metastatic } \\
\text { dissemination }\end{array}$ & $\begin{array}{l}\text { PD at primary site and } \\
\text { new metastatic disease }\end{array}$ \\
\hline Tumor genetics & BRAF V600E & BRAF V600D & KRAS p.G60_Q61ins7 & BRAF V600E & Negative for mutations \\
\hline $\begin{array}{l}\text { MAPK-P inhibition } \\
\text { therapy }\end{array}$ & Dabrafenib for $12 \mathrm{mo}$ & $\begin{array}{l}\text { Dabrafenib for } 7 \mathrm{mo} \text {; } \\
\text { then dabrafenib + } \\
\text { trametinib for } 11 \mathrm{mo}\end{array}$ & $\begin{array}{l}\text { Possible candidate if } \\
\text { disease progresses }\end{array}$ & $\begin{array}{l}\text { Possible candidate if } \\
\text { disease progresses }\end{array}$ & N/A \\
\hline $\begin{array}{l}\text { Response to } \\
\text { targeted therapy }\end{array}$ & $\begin{array}{l}\text { Resolution of } \\
\text { metastatic disease, } \\
\text { decrease in primary } \\
\text { tumor }\end{array}$ & $\begin{array}{l}\text { Stable primary disease } \\
\text { with improvement } \\
\text { in metastatic tumor } \\
\text { burden }\end{array}$ & N/A & N/A & N/A \\
\hline Reported toxicity & Skin rash & Fatigue & N/A & N/A & $N / A$ \\
\hline
\end{tabular}

Abbreviations: CMT, chemotherapy; ETV, endoscopic third ventriculostomy; MAPK-P, mitogen activated protein kinase pathway; N/A, not applicable; PD, progressive disease; RT, radiotherapy; SD, stable disease. 


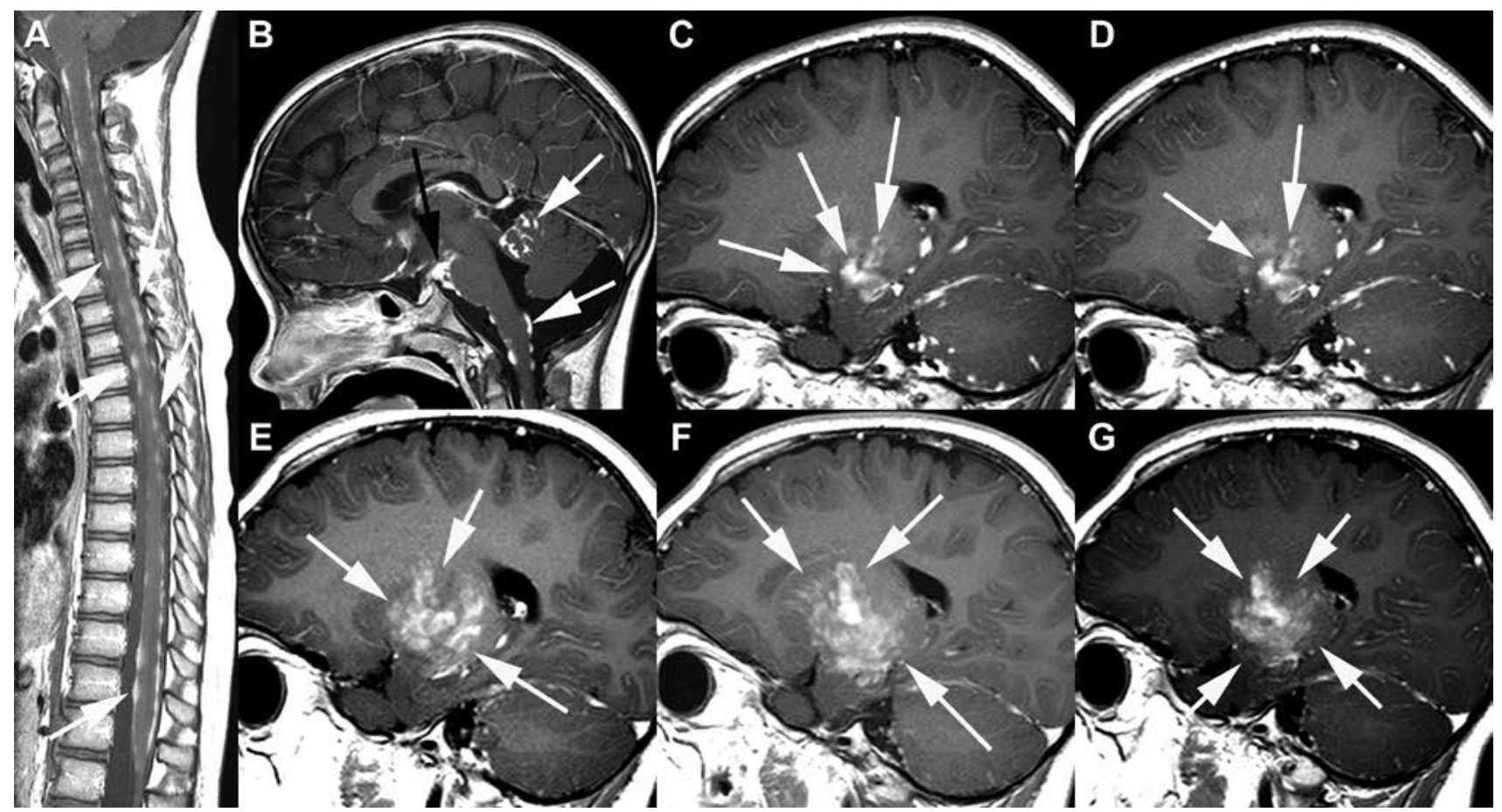

Figure 1. MRI in Patient 2 with BRAF V600D-mutated pilocytic astrocytomas in the optic-hypothalamic region. (A) Sagittal T1-weighted gadoliniumenhanced image of the spine shows extensive thick, nodular leptomeningeal tumor deposits on the spinal cord surface (white arrows). (B-G) Sagittal T1-weighted images of the brain obtained after administration of intravenous gadolinium. (A-D) Images obtained before dabrafenib was started. Black arrow (B) depicts the optic-hypothalamic tumor. There is a leptomeningeal tumor involving the cerebellum and surfaces of the brainstem (white arrows). Sagittal T1-weighted images with gadolinium from 3 months (E) and 6 months (F) post-dabrafenib; these images show progressive increase in tumor burden (white arrows). (G) Sagittal T1-weighted with gadolinium image obtained after 3 months of combination therapy with dabrafenib at $50 \mathrm{mg}$ and trametinib at $0.5 \mathrm{mg}$ daily (9 months of targeted therapy); this shows a decrease in tumor burden.

leptomeningeal disease with stable primary site disease; however, at 6 months of therapy with singleagent dabrafenib, an increase in primary site tumor burden (with stable leptomeningeal disease) was observed on imaging (Figure 1E, F). Subsequently, the MEK inhibitor trametinib was added at $0.5 \mathrm{mg}$ daily, and stable disease was achieved after 3 months of combined dabrafenib and trametinib therapy (Figure $1 G)$. Her most recent MRI after 11 months of combination therapy showed stable disease. The only reported adverse effect was fatigue.

Patient 3 presented at 4 years of age with ataxia, hallucinations, and marked hydrocephalus associated with large midbrain PA and extensive intracranial and spinal leptomeningeal metastatic disease. His treatment regimen included endoscopic third ventriculostomy, chemotherapy (carboplatin + vinblastine; bevacizumab, everolimus), and craniospinal RT with overall stable disease status. The mutation KRAS G60_Q61ins7 was identified in his tumor using the 25-gene test. He is a potential future candidate for targeted therapy if his disease progresses.
Patient 4 presented with precocious puberty and vision concerns at the age of 7 years; he was subsequently diagnosed with large optic PA infiltrating into the thalamus and brainstem. He was treated with chemotherapy (carboplatin + vinblastine) with overall stable disease status. BRAF V600E was identified in the tumor using the 25-gene test, and he may be a future candidate for targeted therapy if his disease progresses.

Patient 5 was diagnosed at 15 years of age with a third-ventricle PA with metastatic disease to the spinal cord. He underwent subtotal surgical tumor resection followed by chemotherapy (carboplatin + vincristine). His follow-up MRI after 3 months of treatment showed interval progression of the primary tumor mass and metastatic disease along ependymal surfaces. Craniospinal RT was recommended given his progressive and metastatic tumor. His tumor tested negative for activating variants by the 25-gene test. Evaluation for the KIAA1549-BRAF fusion is being considered. 


\section{Discussion}

In our study, 4 of 5 patients with disseminated PAs had nonfusion MAPK pathway-activating events, including BRAF V600E, BRAF V600D, and KRAS G60_Q61 ins7. The fifth patient, whose tumor was negative for activating variants, was analyzed using the 25-gene panel test, which does not detect the BRAF fusion. Of the 4 patients who tested positive for an activating event, 3 had an extracerebellar location of the primary tumor in the optic/thalamic region (BRAF V600E), optic/hypothalamic region (BRAF V600D), and mid-brain (KRAS G60_Q61 ins7; Table 1). BRAF V600D has not been previously reported in low-grade gliomas, but is a known activating mutation in melanoma which can be inhibited by dabrafenib. ${ }^{14}$ KRAS G60_Q61ins7 has only been reported once previously in a patient with juvenile myelomonocytic leukemia; this KRAS mutation had in vitro sensitivity to MEK inhibition. ${ }^{15}$

The anatomic location of PAs appears to have an association with the frequency of nonfusion MAPK pathway-activating mutations. In a previous study, BRAF nonfusion mutations were reported more frequently in extracerebellar PAs $(\approx 20 \%)$ compared with cerebellar PAs $(\approx 2 \%){ }^{6}$ In contrast, MAPK pathway-activating gene fusion events have been reported more frequently in cerebellar compared with extracerebellar PAs. ${ }^{3,16}$

In 2013, a whole-genome sequencing study of 96 patients with PAs was reported by the International Cancer Genome Consortium PedBrain Tumor Research Project. ${ }^{16}$ In this study, MAPK pathway activation was identified in all PAs. The most frequent activating events were gene fusions involving $B R A F$ or NTRK2. Nonfusion activating mutations were identified in the BRAF, FGFR1, NF1, KRAS, and PTPN11 genes. A separate whole-genome sequencing study of 39 low-grade gliomas and low-grade glioneuronal tumors similarly identified MAPK pathway-activating mutations in $95 \%$ of cases in a combined category of PAs and pilomyxoid astrocytomas. ${ }^{17}$ Of importance to the present case series, a recent report identified that PAs with dissemination have similar genetic findings to PAs without dissemination. ${ }^{18}$ Unlike the currently presented series of patients, which showed only nonfusion mutations, the prior series of 17 patients with disseminated PAs showed KIAA1549-BRAF fusion in 8 of 12 patients tested $(66.6 \%$; 5 patients could not be tested by fluorescence in situ hybridization); only 1 of the 17 patients had a BRAF mutation, and no patients had mutations in HRAS, KRAS, NRAS, or FGFR1 on targeted analysis. ${ }^{18}$

Frequent MAPK pathway activation through fusion and nonfusion mutations has been recognized as a potential target for inhibition therapy. However, an early preclinical cell line model with the KIAA1549. BRAF fusion demonstrated that treatment with firstgeneration BRAF inhibitors (ie, vemurafenib) may result in paradoxical activation rather than inhibition of MAPK signaling. ${ }^{19}$ The failure of first-generation BRAF inhibitors to inhibit MAPK signaling was hypothesized to be a result of a constitutively activated KIAA1549-BRAF fusion homodimer. In contrast, a second-generation BRAF inhibitor, PLX PB-3, demonstrated inhibition of MAPK signaling from the KIAA1549-BRAF fusion homodimer. ${ }^{19}$ The results of these prior in vitro studies suggest the activity of second-generation BRAF inhibitors and/or downstream MAPK pathway inhibition in the treatment of PAs.

\section{Conclusions}

Our series included 2 cases of pediatric disseminated PAs that had a clinical response to MAPK pathway inhibition. The implications of the findings in this series of patients are that (1) MAPK pathway activation is clearly present in disseminated PAs, and (2) inhibition of the MAPK pathway in select patients may stabilize or decrease disease burden. Future clinical studies of disseminated PAs should incorporate genomic methods that comprehensively analyze the MAPK pathway for activating alterations (eg, single nucleotide, insertion/deletions, and fusions).

\section{References}

1. Johnson KJ, Cullen J, Barnholtz-Sloan JS, et al. Childhood brain tumor epidemiology: a brain tumor epidemiology consortium review. Cancer Epidemiol Biomarkers Prev 2014;23:2716-2736.

2. Penman CL, Faulkner C, Lowis SP, et al. Current understanding of BRAF alterations in diagnosis, prognosis, and therapeutic targeting in pediatric low-grade gliomas. Front Oncol 2015;5:54.

3. Forshew T, Tatevossian RG, Lawson AR, et al. Activation of the ERK/ MAPK pathway: a signature genetic defect in posterior fossa pilocytic astrocytomas. J Pathol 2009;218:172-181. 
Drobysheva et al

4. Jones DT, Kocialkowski S, Liu L, et al. Oncogenic RAF1 rearrangement and a novel BRAF mutation as alternatives to KIAA1549:BRAF fusion in activating the MAPK pathway in pilocytic astrocytoma. Oncogene 2009;28:2119-2123.

5. Miller C, Guillaume D, Dusenbery K, et al. Report of effective trametinib therapy in 2 children with progressive hypothalamic optic pathway pilocytic astrocytoma: documentation of volumetric response. J Neurosurg Pediatr 2016;19:1-6.

6. Schindler G, Capper D, Meyer J, et al. Analysis of BRAF V600E mutation in 1,320 nervous system tumors reveals high mutation frequencies in pleomorphic xanthoastrocytoma, ganglioglioma and extra-cerebellar pilocytic astrocytoma. Acta Neuropathol 2011;121:397-405.

7. Mazloom A, Hodges JC, Teh BS, et al. Outcome of patients with pilocytic astrocytoma and leptomeningeal dissemination. Int J Radiat Oncol Biol Phys 2012;84:350-354.

8. Bautista F, Paci A, Minard-Colin V, et al. Vemurafenib in pediatric patients with BRAF V600E mutated high-grade gliomas. Pediatr Blood Cancer 2014;61:1101-1103.

9. del Bufalo F, Carai A, Figa-Talamanca L, et al. Response of recurrent BRAF V600E mutated ganglioglioma to vemurafenib as single agent. J Transl Med 2014;12:356.

10. Lassaletta A, Guerreiro Stucklin A, Ramaswamy V, et al. Profound clinical and radiological response to BRAF inhibition in a 2-month-old diencephalic child with hypothalamic/chiasmatic glioma. Pediatr Blood Cancer 2016;63:2038-2041.
11. Robinson GW, Orr BA, Gajjar A. Complete clinical regression of a BRAF V600E-mutant pediatric glioblastoma multiforme after BRAF inhibito therapy. BMC Cancer 2014;14:258.

12. Rush S, Foreman N, Liu A. Brainstem ganglioglioma successfully treated with vemurafenib. J Clin Oncol 2013;31:e159-160.

13. Skrypek M, Foreman N, Guillaume D, et al. Pilomyxoid astrocytoma treated successfully with vemurafenib. Pediatr Blood Cancer 2014;61:2099-2100.

14. Gentilcore G, Madonna G, Mozzillo N, et al. Effect of dabrafenib on melanoma cell lines harbouring the BRAF(V600D/R) mutations. BMC Cancer 2013;13:17

15. White $\mathrm{Y}$, Bagchi A, Van Ziffle J, et al. KRAS insertion mutations are oncogenic and exhibit distinct functional properties. Nat Commun 2016;7:10647.

16. Jones DT, Hutter B, Jager N, et al. Recurrent somatic alterations of FGFR1 and NTRK2 in pilocytic astrocytoma. Nat Genet 2013;45:927-932.

17. Zhang J, Wu G, Miller CP, et al. Whole-genome sequencing identifies genetic alterations in pediatric low-grade gliomas. Nat Genet 2013;45:602612.

18. Gessi M, Engels AC, Lambert S, et al. Molecular characterization of disseminated pilocytic astrocytomas. Neuropathol Appl Neurobio 2016;42:273-278.

19. Sievert AJ, Lang SS, Boucher KL, et al. Paradoxical activation and RAF inhibitor resistance of BRAF protein kinase fusions characterizing pediatric astrocytomas. Proc Natl Acad Sci U S A 2013;110:5957-5962.

\section{Role of Biosimilars in Quality Cancer Care}

Understanding the Expanding

Gain expert guidance in integrating biosimilars into your oncology practice, including insights into their development, review of available clinical data, and appraisal of common misconceptions.

Topics include:

- Biosimilars in Oncology: Insights into the Development and Evaluation Process

- Landscape of Biosimilars in Supportive and Therapeutic Cancer Care

- Integrating Biosimilars into Oncology Practice
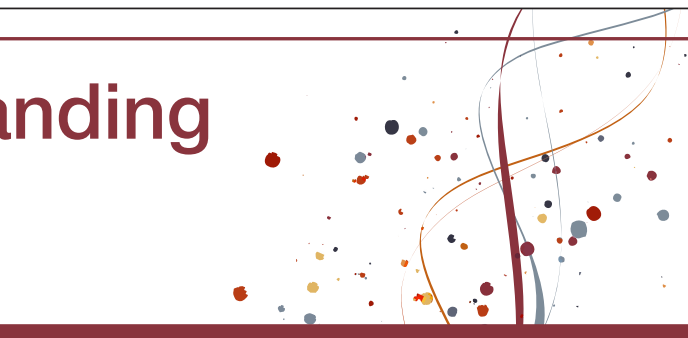

Earn up to 1.5 CME/CE credits! View webcast

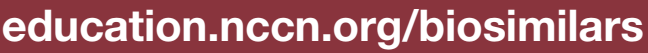

Jointly provided by National Comprehensive Cancer Network ${ }^{\circledast}\left(\mathrm{NCCN}^{\circledR}\right)$ and Clinical Care Options, LLC This program has been approved for AMA PRA Category 1 Credit'TM for physicians and will award contact hours for nurses, pharmacists, and other health care professionals. Complete accreditation information is available online.

Supported by educational grants from Pfizer Inc. and Sandoz Inc., a Novartis Division

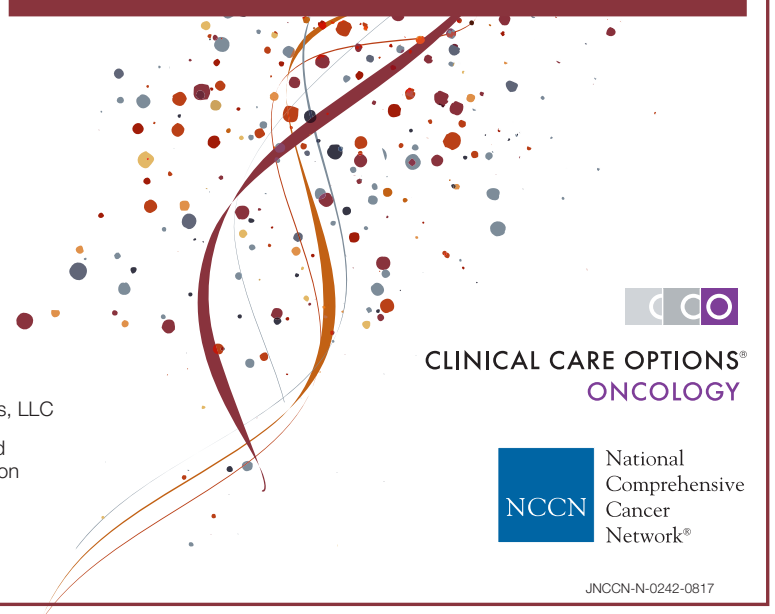

